\title{
Neurologic Late Effects Associated with Radiologic Evidence of Vertebral Osteoradionecrosis after Salvage Laryngectomy: A Syndrome Associated with Survivors of Laryngeal and Hypopharyngeal Cancer
}

Andrew J. Rosko, $M D^{1}$, Matthew E. Spector, $M D^{1}$, Garrett R. Griffin, $M D^{1,2}$, Jeffrey M. Vainshtein, MD ${ }^{3,4}$, Jae Lee, $\mathrm{MD}^{3}$, Carol R. Bradford, MD ${ }^{1}$, Mark E.P. Prince, MD ${ }^{1}$, Jeffrey S. Moyer, $\mathrm{MD}^{1}$, Francis P. Worden, $\mathrm{MD}^{5}$, Avraham Eisbruch, $\mathrm{MD}^{3}$, Douglas B. Chepeha, MD, MSPH ${ }^{1,6}$

1 Department of Otolaryngology-Head and Neck Surgery, University of Michigan, Ann Arbor, MI, USA

2 Midwest ENT Specialists, Woodbury, MN, USA

3 Department of Radiation Oncology, University of Michigan, Ann Arbor, MI, USA

4 Department of Radiation Oncology, Emory University, Atlanta, GA, USA

5 Department of Medical Oncology, University of Michigan, Ann Arbor, MI, USA

6 Department of Otolaryngology-Head and Neck Surgery, University of Toronto, Toronto, ON, Canada

Acknowledgement

of grant support:

None

Financial disclosure: None

Conflict of interest: None

Presentation at meetings: None

Corresponding author: Douglas B. Chepeha, MD, MScPH, FACS, FRCS(C) 200 Elizabeth Street

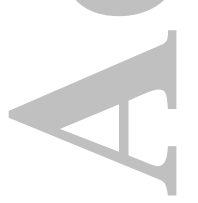
8th Floor $8 \mathrm{NU} 881$

Toronto ON, M5G 2C4 douglas.chepeha@uhn.ca

(416) 946-4629 Phone

(416) 946-2300 Fax

Keywords:

Late Effects, Head and Neck Cancer, Myelopathy, Osteoradionecrosis, Salvage laryngectomy

This is the author manuscript accepted for publication and has undergone full peer review but has not been through the copyediting, typesetting, pagination and proofreading process, which may lead to differences between this version and the Version record. Please cite this article as doi:10.1002/ hed.24348. 


\section{ABSTRACT}

Background: Delayed non-specific posterior neck pain after pharyngeal instrumentation can be associated with a syndrome of rapidly progressive neurologic embarrassment. We present this cohort to help define the syndrome and aid in early detection.

Methods: Retrospective case series of 6 patients presenting from $2003-2012$ with a history laryngeal or hypopharyngeal squamous cell carcinoma (SCC) who underwent radiation (XRT) or chemoradiation (CRT) followed by salvage laryngectomy.

Results: Posterior neck and upper back pain developed a mean of 27.5 days after instrumentation of the pharynx (reconstruction after laryngectomy or pharyngeal dilation). Myelopathy developed an average of 21.5 days after onset of posterior neck pain. Five patients required urgent decompression. Three patients developed quadriplegia. The disease specific mortality was $50 \%$.

Conclusion: There is a syndrome of late neurological effects after radiation, salvage surgery and pharyngeal instrumentation that is associated with high morbidity and mortality. 


\section{INTRODUCTION}

Since the Veterans Affairs' (VA) laryngeal cancer study was published in 1991, there have been an increasing number of patients with stage III and IV laryngeal and hypopharyngeal squamous cell carcinoma (SCC) being treated with chemoradiation (CRT). The major advantage of this treatment approach is preservation of the larynx in $2 / 3$ of the surviving patients during the first 5 years post treatment. Preservation of the larynx is a clear advantage, but for some patients there are unpredictable late effects. ${ }^{1}$ While radiation therapy (XRT) and CRT provide a chance for organ preservation, treatment has deleterious effects on adjacent normal tissue, as it induces a hypoxic, hypocellular, and hypovascular environment. ${ }^{2}$ The tissue effects are the most pronounced for patients who undergo primary XRT or CRT and require salvage surgery for persistent or recurrent disease. This study attempts to describe the syndrome of subtle posterior neck and upper back pain in patients after surgical instrumentation of their pharynx with a history of XRT who progress to myelopathy with radiologic evidence of spinal epidural thickening and loss of fat signal in the marrow space of the cervical vertebrae compatible with cervical vertebral osteoradionecrosis (ORN).

\section{MATERIALS AND METHODS}

\section{Study Design}

A retrospective case series of 6 patients who developed a syndrome of nonspecific posterior neck pain localized to the vertebrae with neurologic symptoms of myelopathy and radiologic evidence of vertebral ORN was conducted. To evaluate the 
characteristics of this syndrome, a retrospective chart review was performed at University of Michigan for patients presenting between 2003 and 2012.

\section{Eligibility Criteria}

Patients were eligible for inclusion in the cohort if they had a history of SCC of the larynx or hypopharynx treated with curative radiation with or without chemotherapy and subsequently developed a syndrome of subtle pain in the posterior neck and upper back 2-8 weeks after instrumentation of the pharynx associated with symptoms of myelopathy and radiologic evidence of spinal epidural thickening and loss of fat in the marrow space of the cervical vertebrae compatible with cervical vertebral ORN.

\section{Initial Presentation and Treatment}

Patient demographics and oncologic treatment are shown in Table 1 and Table 2. When initially diagnosed with SCC of the larynx or hypopharynx, half of the patients presented with Stage II disease and the other half presented with stage IV disease. Of the 5 patients who underwent chemotherapy 2 patients received carboplatin alone, 1 received carboplatin and 5-fluorouracil, 1 received carboplatin and paclitaxel and 1 received received 5-fluorouracil, docetaxel and hydroxyurea. The remaining patient received amifostene. The average radiation delivered to the primary site was $71 \mathrm{~Gy}$ (range 68-75). One patient received BID fractionation and had 5 weeks of treatment. The remaining patients were treated with daily fractionation over 7 weeks. Two patients received radiation at the University of Michigan with the remainder being treated at other institutions. Radiation fields were not available in two patients treated at outside 
institutions. The mean and maximum doses to the cervical vertebral bodies (C4-C6) were 54.4 Gy and 69.8 Gy, respectively.

\section{Salvage Surgery}

The mean interval between XRT and salvage surgery was 25.9 months with a range of $11.6-70.8$ months. Four out of six (67\%) patients underwent total laryngectomy and $2 / 6(33 \%)$ patients underwent a total laryngopharyngectomy. All patients underwent autogenous tissue transplantation for reconstruction and cricopharyngeal myotomy for voicing with the immediate placement of a nasogastric tube for nutrition. All patients underwent delayed tracheal-esophageal puncture.

\section{Statistical Analysis}

Variables under study included age, sex, tumor stage, timing of XRT, chemotherapeutic regimen, indication for salvage surgery, surgical defect, and reconstructive approach. Variables specific to the syndrome were collected including instrumentation of the pharynx, presenting symptoms, interval to diagnosis, microbiological culture, alcohol use, tobacco use, BMI, TSH and albumin. Specific outcomes of interest included neurologic disability and recovery after treatment. The date of instrumentation of the pharynx was defined as the most recent pharyngeal dilation (in the case of 4 patients) or reconstruction of the pharynx after surgical salvage (in the case of 2 patients) prior to the development of the syndrome. The date of symptom onset was defined as the first date that patients experienced neck or high back pain. The date of radiologic evidence of vertebral ORN diagnosis was the date 
that cross sectional imaging was obtained showing spinal epidural thickening and loss of fat in the marrow space of the cervical vertebrae. Microbial culture data was available in all patients. Antibiotic regimen was tabulated. Disease specific survival was determined from the date of imaging showing loss of fat in the marrow space of the vertebral body. Univariate statistics were calculated including mean and range. This study received University of Michigan Institutional Review Board Approval (IRB).

\section{RESULTS}

\section{Summary of the Syndrome}

The syndrome includes non-specific symptoms of posterior neck pain localized to the vertebrae with neurologic symptoms of myelopathy and radiologic evidence cervical dural thickening and loss of fat in the marrow space of the cervical vertebrae compatible with a radiologic diagnosis of vertebral ORN that is culture positive for salivary organisms. This developed in 6 patients with a history of SCC of the larynx or hypopharynx treated with XRT with or without chemotherapy followed by salvage laryngectomy. Symptoms of the syndrome developed a mean of 27.5 days after instrumentation of the pharynx (reconstruction after laryngectomy or pharyngeal dilation). Neurologic symptoms of myelopathy developed an average of 21.5 days after symptom onset, which prompted cross sectional imaging. Most patients required surgical decompression and stabilization of the cervical spine. All patients underwent a prolonged course of broad-spectrum antibiotics. Disease specific mortality was $50 \%$ (Figure 1). 


\section{Instrumentation of the Pharynx}

In this cohort, 4/6 (67\%) patients presented with the syndrome after dilation for pharyngeal stenosis and 2/6 (33\%) presented after developing either a fistula or dehiscence of the closure while healing from pharyngeal reconstruction following salvage laryngectomy. For those undergoing dilation, the mean interval between salvage laryngectomy with pharyngeal reconstruction and the inciting instrumentation of the pharynx was 53.5 months with a range of 20.9 months to 83.9 months. There were no frank perforations during the dilation of any patient. For the 6 patients, the mean interval from instrumentation (dilation or reconstruction) of the pharynx to the development of symptoms was 27.5 days with a range of 9 to 56 days.

\section{Presenting Symptoms and Signs}

All patients initially presented with posterior neck pain localized to the vertebrae and $2 / 6(33 \%)$ of patients reported a concurrent headache. The mean interval between the onset of neck and back pain and subsequent development of myelopathy was 21.5 days with a range of 1-65 days. Symptoms and signs of myelopathy included isolated bowel incontinence $(1 / 6,17 \%)$, upper extremity weakness and paresthesias $(1 / 6,17 \%)$ lower extremity weakness and paresthesias (1/6, 17\%), hemiplegia (Brown-Sequard) $(1 / 6,17 \%)$ or quadriplegia $(2 / 6,33 \%)$. Fifty percent of patients were febrile at presentation and all but one had a leukocytosis. Mean white blood cell count was 13.7 (range 6.1-17.9). Four out of 6 patients were tachycardic at presentation with 2/6 being hypotensive. Eighty seven percent (5/6) of patients were hyponatremic at the time of diagnosis. ESR (mean 83, range 37-122) and CRP (mean 8.3, range 1.8-15.6) were 
elevated in all patients (Table 3). Cross sectional imaging showed loss of fat signal in the marrow space of the cervical vertebrae, myelitis of the adjacent spinal cord and thickening and enhancement of the dura. Five out of six (83\%) patients had evidence of an epidural fluid collection. The epidural fluid collection was a very subtle finding on CT and appeared to be a thickening of the epidural tissues. Five out of six $(83 \%)$ patients required an MRI scan (Figure 2).

\section{The Acute Neurosurgical Management}

All but 1 patient required emergent decompression by neurosurgery within 24 hours of diagnosis. The remaining patient had isolated bowel and bladder incontinence with hyperreflexia in the lower extremities without evidence of an epidural fluid collection. In the remaining 5 patients, 1 underwent laminectomy with drainage of the epidural abscess, 1 underwent laminectomy with posterior fusion, and 3 underwent laminectomy, corpectomy with anterior and posterior fusion. Bone and tissue biopsies were taken in three of the six (50\%) patients, all of which showed bone and soft tissue necrosis and fibrosis, consistent with verterbral ORN. Biopsies were not taken in the remaining three patients. Three patients required more than 1 operation with one patient with the syndrome undergoing 10 operations including creation of a diverting pharyngostome due to recurrent infections. This patient developed a meningocoele and CSF leak leading to recurrent meningitis requiring a deltopectoral flap, pectoralis flap and tensor fascia lata graft for closure.

\section{Microbiology}


Tissue cultures were obtained from all patients. Open cultures were obtained from the bone (1/6) and the epidural space (4/6) in 5 patients and a percutaneous culture from the disc space was obtained from the remaining patient. All but 1 patient had a polymicrobial infection and all organisms cultured were consistent with contamination from the pharynx. Again, it is important to note that in $4 / 6$ patients there was no associated disruption of the visceral space. 4/6 patients grew Streptococcal species in their cultures. Isolated organisms included Streptococcus viridans, Group B streptococcus, Streptococcus milleri, Haemophilus influenza, Klebsiella, Methicillin sensitive and Methicillin resistant Staphylococcus aureus, Pseudomonas aeruginosa, Enterococcus, Proteus vulgaris, Saccharomyces cerevisiae, Candida, Citrobacter freundi, Fúsobacterium nucleatum, and anerobes, not otherwise specified. Patients were treated with broad-spectrum IV antibiotics.

\section{Other Risk Factors}

At the time patients presented with this syndrome there were no current alcohol users and only $1 / 6(17 \%)$ was a current smoker. BMI did not appear to infer extra risk for vertebral ORN as only 1 patient $(17 \%)$ was underweight at the time of salvage laryngectomy and 1 patient was underweight at the time of developing neurological sequela. All but 2 patients had an albumin of 3.5 or greater at the time of ORN diagnosis. All patients were euthyroid (mean TSH 2.86, range $0.47-5.5$ ) at the time of salvage laryngectomy, however $50 \%$ of patients $(3 / 6)$ were found to be hypothyroid at the time of development of neurological sequela (mean 8.36 , range $1.5-13.75$ ). 


\section{Outcome}

Of the 6 patients, 3 patients had no improvement in their neurologic status despite treatment. Two patients had improvement in their neurologic status. One patient who initially presented with Brown-Sequard syndrome progressed to quadriplegia. Half of the patients $(3 / 6)$ succumbed to pneumonia secondary to their neurological debilitation, 2 patients in the acute setting and 1 patient several months after the diagnosis of this syndrome. These 3 patients all had quadriplegia at the time of death. One patient died of widely metastatic disease. The remaining 2 patients continue to be seen in our clinic.

\section{DISCUSSION}

Although there are case reports, this is the first series in the literature of patients with a history of SCC of the larynx or hypopharynx describing this syndrome presenting with subtle neck pain progressing to neurological sequela. ORN of the cervical spine was first reported by Lim et al in 1999 and is a rare complication of XRT. ${ }^{3}$ There have been several case reports in the literature with patients developing ORN of the cervical spine after XRT for head and neck malignancies, though most of these cases occurred in patients treated for nasopharyngeal carcinoma. ${ }^{4-6}$

The pathogenesis of this neurological late effect is poorly understood though several mechanisms have been proposed. ${ }^{7}$ Radiation therapy induces an endarteritis that results in tissue hypoxia, hypocellularity, and hypovascularity with resultant ischemic necrosis of the local tissues including bone..$^{-11}$ In addition, salvage surgery after radiation likely causes additional de-vascularization of the local tissues despite the 
use of free tissue transplantation in these patients. Fortunately, with the advent of IMRT, there is reduced radiation to surrounding normal tissues, including the vertebrae.

For the development of the observed neurological late effects, bacterial translocation was a critical event leading to contamination and infection of the epidural space. The exact mechanism of bacterial spread is unknown, however we speculate that bacteria are seeded via venous hematogenous spread from the pharynx to the venous plexus around the cervical spine. This speculation is supported by the radiologic finding of cord infarction in several patients that was presumably secondary to infectious thrombophlebitis. In addition in 4 of the patients there was no mucosal disruption and no clear communication with the vertebrae and particularly the epidural space.

It is concerning that the presenting symptoms are subtle and non-specific, and once neurological findings are present the progression is rapid and potentially lethal. All of the patients in this study described non-specific posterior neck and upper back pain localizing to the vertebrae. Most patients also described a posterior headache. Neck pain is a common complaint in patients with head and neck cancer, however, this pain is typically located in the anterior or lateral neck and is most often characterized as burning, tènder, sore or throbbing. ${ }^{12}$ In our patients, however, the pain was localized to the posterior neck and back. Though these are fairly non-specific symptoms, physicians need a high level of suspicion in at risk patients with new pain in the spine after any instrumentation of the pharynx. This should prompt a thorough neurologic history and examination to evaluate for subtle signs of early myelopathy such as weakness, numbness, hyperreflexia, paresthesias and bowel/bladder incontinence as this may 
herald pending neurologic embarrassment. Elevation in the ESR and CRP was a sensitive finding in our cohort. Signs of a systemic inflammatory response may also be present.

Cross sectional imaging should be obtained in any patient who has posterior neck pain with a history of CRT and salvage laryngectomy who has undergone instrumentation of the pharynx within the last 8 weeks. A CT scan can be used as a helpful screening tool to identify loss of fat in the marrow space of the cervical vertebrae. If loss of fat is present in the cervical vertebrae, an MRI should be emergently performed and neurosurgery should be consulted for possible emergent decompression to avoid severe neurological sequela. Raza et al. presented a case report in which a patient previously treated with CRT for SCC of the larynx was seen with neck pain and a headache prompting cross-sectional imaging. The patient did not have any symptoms of myelopathy. The patient underwent occipito-cervical fusion and long term neurologic sequela were avoided, highlighting the need for prompt recognition and early intervention. ${ }^{13}$ Similarly, a case was reported by Powell et al in which a patient with radiologic evidence of vertebral ORN was stabilized using a free fibular reconstruction prior to the development of neurologic symptoms, with no resultant long term myelopathy. ${ }^{14}$

Antibiotics targeted at pharyngeal organisms should be initiated empirically and expeditiously. A multidisciplinary approach including infectious disease, neurosurgery, and head and neck surgery are critical to ensure the best possible outcome. Even with aggressive therapy the prognosis is poor with a $50 \%$ disease specific mortality in our cohort. Of the remaining 3 patients, 2 patients (33\%) did have some improvement in 
their overall neurologic status and one patient had stable neurologic disability. This is concordant with the previously reported cases of vertebral ORN in patients treated for SCC of the larynx or hypopharynx. In our review of the literature, we found 6 previously reported cases. ${ }^{13-18,19}$ Collating the outcome of these 6 previously reported cases in the literature, 2 were intervened upon prior to the development of neurologic sequela, 1 patient had persistent, though improved weakness, 1 survived with quadriplegia and 2 patients died with quadriplegia secondary to pneumonia.

This syndrome is important to understand. The patients who present with this syndrome are usually well known to the oncology service. They have undergone multiple uneventful dilations and have a delayed onset of vague posterior neck pain that has been present for days to a few weeks. The last dilation was usually uneventful and there is no difficulty with voicing or swallowing. These vague symptoms in a trusting doctor patient relationship can be observed but not acted upon. Unfortunately once the neurological signs present the timeline becomes very rapid. The neurologic signs are difficult to resolve and the outcome for the patient is not desirable. Because this is not a well-recognized late effect of the treatment of laryngeal or hypopharyngeal cancer it can be difficult to; first identify, and second to motivate the multidisciplinary team to act quickly and aggressively. Neurosurgery needs to be encouraged to approach posteriorly through non-radiated tissue and not to wait to see if antibiotics will reverse neurological signs.

Perhaps a nomenclature would be beneficial for late effects involving specific tissues and sites. The involved structure would be named first, the disease second (ORN), the level of causality and the primary site of the cancer. For example: VORNALC, Vertebral 
Osteoradionecrosis Associated Laryngeal Cancer. This nomenclature has the breadth to be used across involved structure, disease process, causality and primary cancer site.

\section{CONCLUSION}

Initial symptoms are usually non-specific and include posterior neck and back pain as well as headache that develops an average 27.5 days after either dilation or salvage laryngectomy (range 9-56 days) with the radiological diagnosis of cervical dural thickening and loss of fat signal in the marrow of the cervical vertebrae being made on average 21.5 days after the onset of pain (range 1-65 days). The time to diagnosis can be significantly shortened by increased level of suspicion. Elevation in the ESR and CRP was a sensitive finding in our cohort. Many patients presented with signs of a systemic inflammatory response such as fever, tachycardia or hypotension. In the event of epidural fullness patients are treated with emergent surgical decompression to minimize the progression of neurologic deficits and to offer the best chance of recovery. All patients should be cultured and started on broad-spectrum antibiotics. IV antibiotic course was continued for a minimum of 8 weeks. In our series, a multidisciplinary approach was used with head and neck surgery, neurosurgery and infectious disease doctors all playing a pivotal role. The prognosis for these patients is grim with the majority of patients failing to recover. The disease specific mortality in our series was $50 \%$ with the patients with the most severe neurologic deficits succumbing to pneumonia. In the future, early recognition of the importance of subtle posterior neck and upper back pain should decrease mortality and morbidity. 


\section{(1) \\ REFERENCES}

1. Induction chemotherapy plus radiation compared with surgery plus radiation in patients with advanced laryngeal cancer. The Department of Veterans Affairs Laryngeal Cancer Study Group. N Engl J Med 324:1685-90, 1991

2. Marx RE: Osteoradionecrosis: a new concept of its pathophysiology. J Oral Maxillofac Surg 41:283-8, 1983

3. Lim AA, Karakla DW, Watkins DV: Osteoradionecrosis of the cervical vertebrae and occipital bone: a case report and brief review of the literature. Am J Otolaryngol 20:408-11, 1999

4. Chung SJ, Wan-Teck L, Tham SC, et al: Osteoradionecrosis of the cervical spine complicated by pneumocephalus and meningitis in a nasopharyngeal cancer patient radically treated with radiotherapy 11 years ago. BMJ Case Rep 2009, 2009

5. King AD, Griffith JF, Abrigo JM, et al: Osteoradionecrosis of the upper cervical spine: MR imaging following radiotherapy for nasopharyngeal carcinoma. Eur J Radiol 73:629-35, 2010

6. Cheung JP, Wei WI, Luk KD: Cervical spine complications after treatment of nasopharyngeal carcinoma. Eur Spine J 22:584-92, 2013 
7. Jacobson AS, Buchbinder D, Hu K, et al: Paradigm shifts in the management of osteoradionecrosis of the mandible. Oral Oncol 46:795-801, 2010

8. Marx RE: A new concept in the treatment of osteoradionecrosis. J Oral Maxillofac Surg 41:351-7, 1983

9. Bras J, de Jonge HK, van Merkesteyn JP: Osteoradionecrosis of the mandible: pathogenesis. Am J Otolaryngol 11:244-50, 1990

10. Ruggiero SL, Mehrotra B, Rosenberg TJ, et al: Osteonecrosis of the jaws associated with the use of bisphosphonates: a review of 63 cases. J Oral Maxillofac Surg $62: 527-34,2004$

$r$

11. Reuther T, Schuster T, Mende $U$, et al: Osteoradionecrosis of the jaws as a side effect of radiotherapy of head and neck tumour patients--a report of a thirty year retrospective review. Int J Oral Maxillofac Surg 32:289-95, 2003

12. Epstein JB, Wilkie DJ, Fischer DJ, et al: Neuropathic and nociceptive pain in head and neck cancer patients receiving radiation therapy. Head Neck Oncol 1:26, 2009

13. Raza A, Islam M, Lakshmanan P: Secondary atlanto-odontoid osteoarthritis with osteoradionecrosis of upper cervical spine mimicking metastasis. BMJ Case Rep 2013,2013

14. Powell DK, Jacobson AS, Kuflik PL, et al: Fibular flap reconstruction of the cervical spine for repair of osteoradionecrosis. Spine J 13:e17-21, 2013 
5. Donovan DJ, Huynh TV, Purdom EB, et al: Osteoradionecrosis of the cervical spine resulting from radiotherapy for primary head and neck malignancies: operative and nonoperative management. Case report. J Neurosurg Spine 3:159-64, 2005

6. Kosaka Y, Okuno Y, Tagawa $\mathrm{Y}$, et al: Osteoradionecrosis of the cervical vertebrae in patients irradiated for head and neck cancers. Jpn J Radiol 28:388-94, 2010

7. van Wyk FC, Sharma MP, Tranter R: Osteoradionecrosis of the cervical spine presenting with quadriplegia in a patient previously treated with radiotherapy for laryngeal cancer: a case report. J Med Case Rep 3:7262, 2009

8. Prasad KC, Prasad SC, Mouli N, et al: Osteomyelitis in the head and neck. Acta Otolaryngol 127:194-205, 2007

9. Ekbom DC, J DE, Isaacson B, et al: Spinal epidural abscess after cervical pharyngoesophageal dilation. Head Neck 27:543-8, 2005 


\section{(1)}

\section{FIGURE LEGEND}

Figure 1. Timeline of patient's disease progression.

Note intervals in blue measured in months, intervals in red measured in days. Pt 1, 4 and 5 died of pneumonia as a complication of ORN. Pt 2 and 3 are still alive. Pt 6 died of metastatic disease.

Figure 2. Representative MRI from selected patient.
A. T1 weighted sagittal image demonstrating the loss of marrow signal in the vertebral marrow space. B. T2 weighted sagittal image showing the epidural thickening and fluid collection.

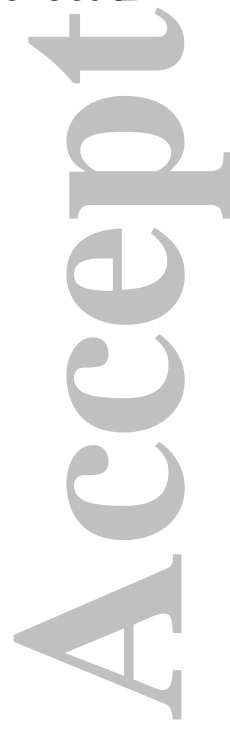




\section{TABLES}

\section{Table 1. Patient Characteristics at Laryngectomy.}

Characteristics

Sex Male Female

Age, Mean (range)

BMI, Mean (range)

TSH, Mean (range)

Albumin, Mean (range)

Diabetes

Tobacco Use

Current

Former

Never

Alcohol Use

Current

Former

Subsite

Never

Supraglottis

Glottis

Subglottis

Piriform sinus

T Classification
T1
T2
T3

T4

N Classification

$$
\text { No }
$$

N1

N2a

$\mathrm{N} 2 \mathrm{~b}$

N2c

N3

Radiation Dose, Mean (range)

Indication for Laryngectomy

Recurrence

Chondroradionecrosis

Surgical Defect

Total laryngectomy

Total laryngopharyngectomy

Cricopharyngeal myotomy

Tracheoesophageal Puncture

Autologous Tissue for Reconstruction

Radial Forearm

Anterolateral Thigh

Lateral Arm

Number of Patients

$50 \%(3)$

$50 \%(3)$

$60.6(55-66)$

$23.2(17.8-33.5)$

$2.86(0.47-5.5) \mathrm{MD}=1$

$3.45(1.3-4.3) \mathrm{MD}=2$

$17 \%(1)$

$67 \%(4)$

$33 \%(2)$

$17 \%(1)$

$50 \%(3)$

$33 \%(2)$

$17 \%$ (1)

$33 \%(2)$

$17 \%(1)$

$33 \%(2)$

$50 \%(3)$

$33 \%(2)$

$17 \%(1)$

$67 \%(4)$

$17 \%(1)$

$17 \%(1)$

$-$

71 Gy (68-75 Gy)

$83 \%(5)$

$17 \%(1)$

$67 \%(4)$

$33 \%(2)$

$100 \%(6)$

$100 \%(6)$

$50 \%(3)$

$33 \%(2)$

$17 \%(1)$

John Wiley \& Sons, Inc.

This article is protected by copyright. All rights reserved. 


\section{Table 2. Patient Characteristics at Vertebral ORN Diagnosis.}

Characteristics

Age, Mean (range)

BMI, Mean (range)

TSH, Mean (range)

Albumin, Mean (range)
Number of Patients

$63.7(56.8-73.0)$

$23.6(17.6-30.8)$

$8.36(1.5-13.75)$

$3.5(3.0-4.2)$

\section{Table 3. Presenting symptoms and signs.}

\section{Signs and Symptoms}

Headache

Posterior neck or upper back pain

Upper extremity weakness/numbness

Lower extremity weakness/numbness

Isolated Urinary/Bowel dysfunction

Hemiplegia

Quadriplegia

Fever

Tachycardia

Hypotension

Hyponatremia

Leukocytosis

Elevated Erythrocyte Sedimentation Rate

Elevated C-Reactive Protein
Number of Patients

$33 \%(2 / 6)$

$100 \%(6 / 6)$

$17 \%(1 / 6)$

$17 \%(1 / 6)$

$17 \%(1 / 6)$

$17 \%(1 / 6)$

$33 \%(2 / 6)$

$50 \%(3 / 6)$

$67 \%(4 / 6)$

$33 \%(2 / 6)$

$83 \%(5 / 6)$

$83 \%(5 / 6)$

$100 \%(5 / 5)^{*}$

$100 \%(3 / 3)^{* *}$

* Lab not ordered at presentation for 1 patient.

${ }^{* *}$ Lab not ordered at presentation for 3 patients. 
Table 4. Proposed protocol for the diagnosis and treatment of late neurological effects associated with radiologic evidence of vertebral osteoradionecrosis.

\section{Vertebral Osteoradionecrosis Protocol}

1. Maintain a high index of suspicion in patients that present with new posterior neck, or back pain with or without headache in the context of recent instrumentation of the pharynx or mucosal disruption.

2. Obtain vital signs including temperature, blood pressure and heart rate as well as labs including a basic metabolic panel to assess for hyponatremia, complete blood count with differential to assess for leukocytosis along with ESR and CRP.

3. Due to immediate availability of CT with contrast, obtain a screening CT.

4. Confirm the diagnosis with MRI if questions persist.

5. Obtain early Neurosurgery consultation at the same time as imaging is requested for possible urgent stabilization, abscesses drainage and cultures. Use a posterior approach for the drainage.

6. Promptly begin empiric broad-spectrum antibiotics with consultation from Infectious Disease.

7. Admit the patient to the hospital for frequent neurologic exams.

8. Tailor antibiotics to culture results if available.

9. Perform pharyngeal diversion to prevent further salivary soiling of vertebrae if necessary. 


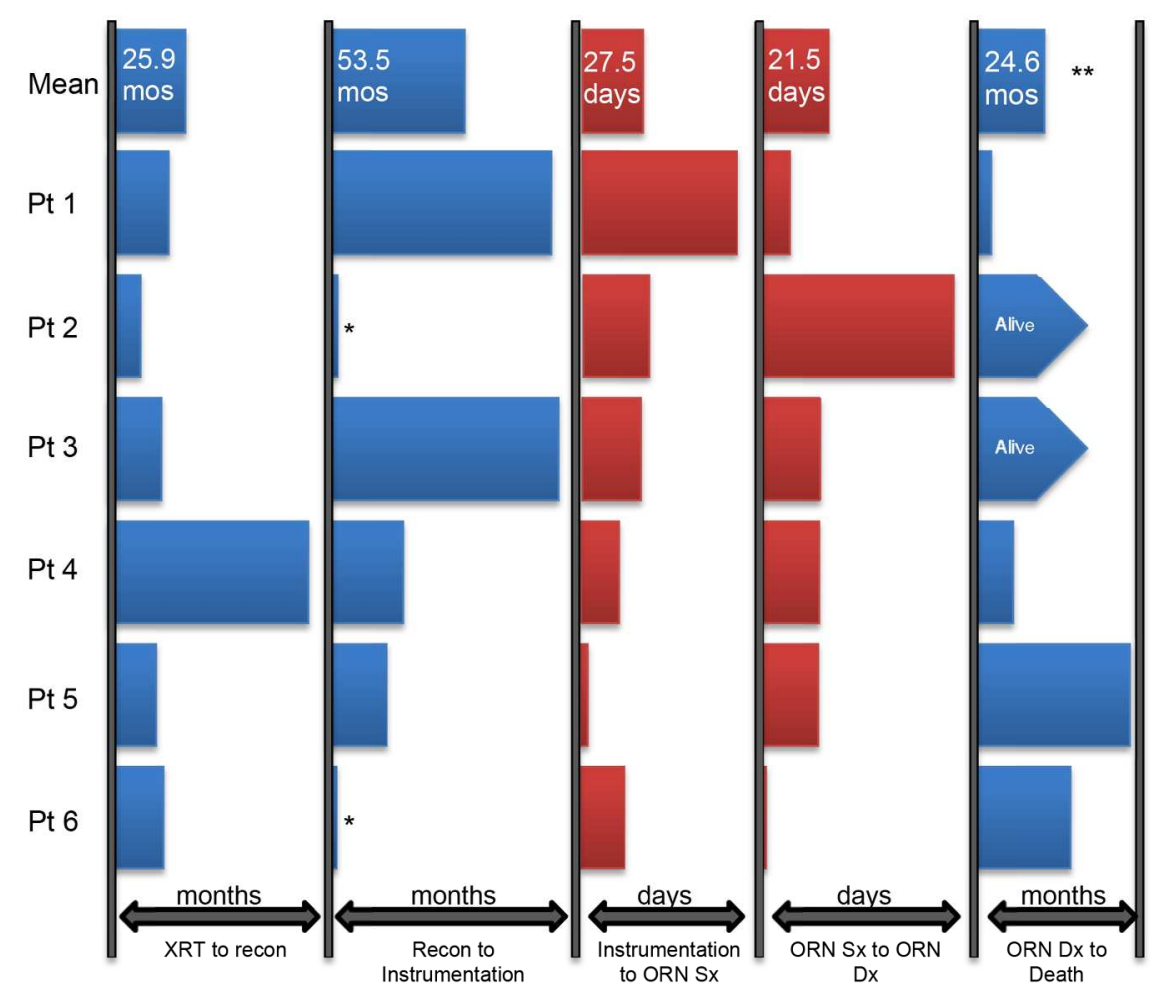

* Indicates patients whose instrumentation of the pharynx was their initial reconstruction and did not have pharyngeal dilation. Mean interval from reconstruction to instrumentation is calculated excluding these 2 patients.

** Mean interval to death presented in patients who died as complications of vertebral ORN.

Figure 1. Timeline of patient's disease progression.

Note intervals in blue measured in months, intervals in red measured in days. Pt 1, 4 and 5 died of pneumonia as a complication of ORN. Pt 2 and 3 are still alive. Pt 6 died of metastatic disease.

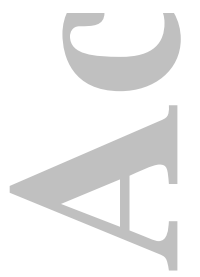
$176 \times 177 \mathrm{~mm}(300 \times 300 \mathrm{DPI})$ 


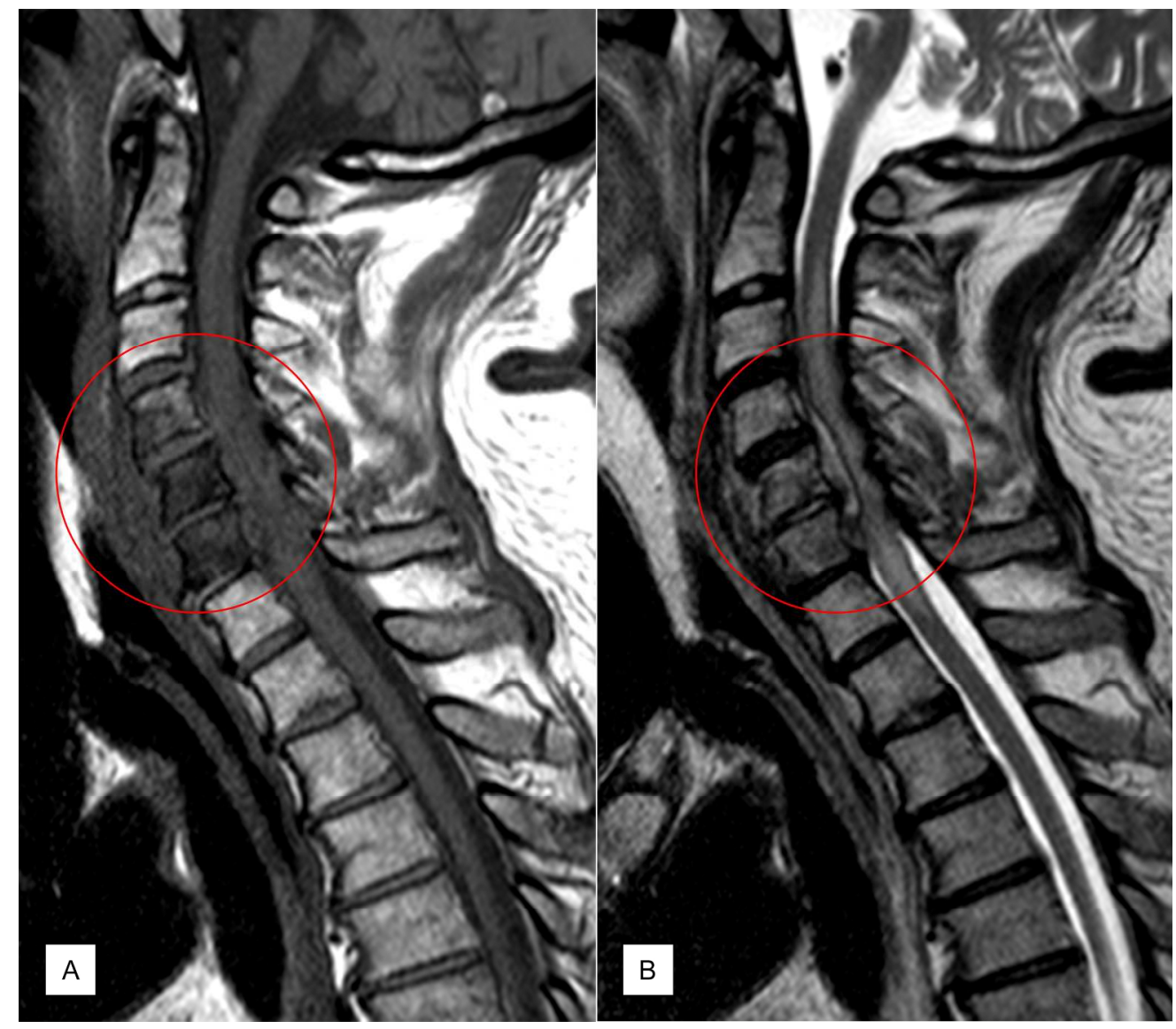

Figure 2. Representative MRI from selected patient.

A. T1 weighted sagittal image demonstrating the loss of marrow signal in the vertebral marrow space. B. T2 weighted sagittal image showing the epidural thickening and fluid collection.

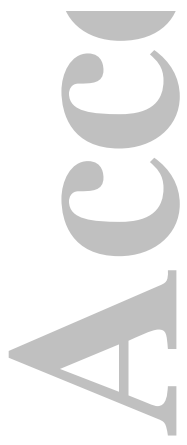

$212 \times 186 \mathrm{~mm}(300 \times 300 \mathrm{DPI})$

John Wiley \& Sons, Inc.

This article is protected by copyright. All rights reserved. 